\title{
The comparative study of equal channel angle and step angle of the buckle battery sealing concave film
}

\author{
Wenhuan GAO, Huilai SUN \\ College of Mechanical Engineering, Tianjin Polytechnic University, Tian jin China, 300387 \\ *344420538@qq.com
}

Keywords: Button cell; Sealing concave film; Contact analysis; ABUQU

\begin{abstract}
Based on the 3D modeling of closing machine by SolidWorks, the sealing process of button battery is mainly analyzed and comparative study on equal channel angular sealing concave film and the sealing step angle concave film are studied with the contact stress with battery with the change of time. In this paper, the finite element model and the analysis of the interference in the ABUQUS are established and the contact deformation of the positive shell of a button cell in a sealing period is obtained. The relationship between the radius of the sealing film and the contact stress of the battery positive shell is analyzed which provide reference value for the production and manufacture of the button cell sealing machine.
\end{abstract}

\section{Introduction}

With the development of the battery industry, the button battery plays a more and more important role in the battery industry. Some batteries are used for toys and gifts others are used in the computer motherboard CMOS battery. [1][2] There are many factors which influence the battery after stamping sealing side of high, such as die stamping springback sealing chamfering, etc.[3]In order to get the high consistency of the battery, it is necessary to reduce the springback.Part type surface springback is one of the most simple springback problems. [4]

The key of springback compensation is the size of the amount of compensation. In the creation of the surface with line, the surface is compensated for the curved surface, which can be designed with the radius of the curved surface, so as to reduce the amount of springback.[5]

\section{Design of double power cam.}

Structure design and analysis of sealing concave membrane.First of all,we need to determine the value of the sealing concave film rounded, and now the company generally uses the $\mathrm{R}=1$.So the radius of the radius should be in the vicinity of $\mathrm{R}=1$. Under other assumptions, the numerical value of the $\mathrm{R}=1$ and $\mathrm{R}=0.9$ of the two corners is assumed. The CR2032 cell model is shown ss shown in Figure 3 and the base sealing sealing membrane $R=1, R=0.9$ diameter concave.[6]

\section{The simulation based on ABUQUS}

Description of the process. The battery is assumed to be $10 \mathrm{kN}$ pressure and the pressure of the 
battery is pressed into the sealing cavity. The sealing concave film is fixed, the other surface has no contact and the friction coefficient of the contact surface is 0.2. Material characteristics of the battery and the sealing film: $E=210000 \mathrm{MPa}, \mu=0.3$ 。

Comparasion of simulation analysis under ABUQUS.In the ABUQUS, a two dimensional model of CR2032 battery was established, and the two dimensional model of equal channel angular sealing concave film. Because the model is symmetric, the 1/2 model is used. After that, the parts, material and section properties were created.

Define the assembly and division of the grid, then set the analysis step, define the contact, and define the boundary conditions which can be submitted after the analysis of the job. [7]

Analysis results are as follows:

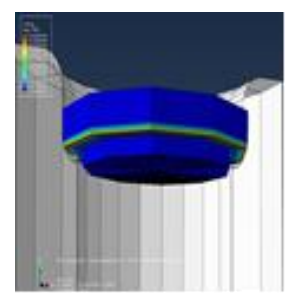

Figure 13D cloud image

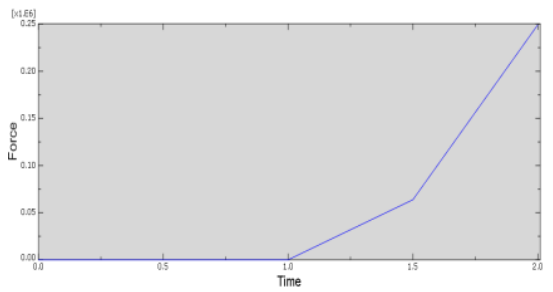

Figure 2 The relationship of contact and time

It is concluded that the maximum stress at this moment is $\mathrm{MPa} 4.689 \mathrm{e}+04$.

Comparative analysis of the step angle concave sealing film is made in this paper. Two-dimensional model of two-dimensional model and variable battery built in CR2032 ABUQUS in the film sealing concave angle are analyzed. Analysis results are as follows:

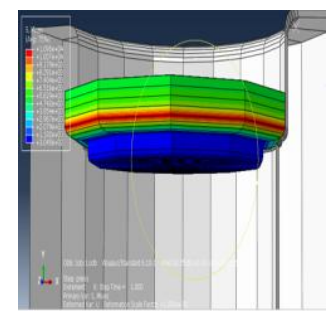

Figure3 3Dcloudimage

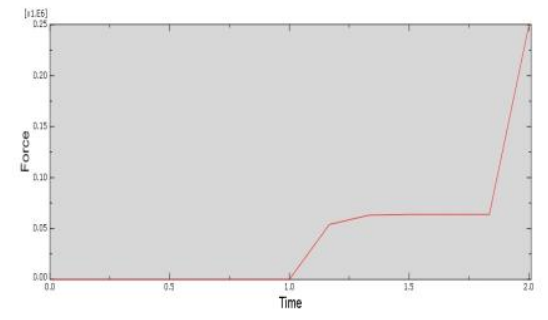

Figure4 Contact force and time

It is concluded that the maximum stress at this moment is MPa $1.095 \mathrm{e}+04$.

It can seen through the comparative analysis of stress contours of equal channel angular diameter and angle of concave concave membrane membrane.

\section{Experimental study}

In the actual production, the total height and the edge of the battery were measured, so as to determine the deviation between the theoretical height, so as to ensure the consistency of the battery, which can achieve the purpose of improving the quality of the battery.Then analyze and determine the value of equal channel angular sealing film and the sealing concave concave angle adjustable high and high total film edge consistently higher.The median interval $2.5 \mathrm{~mm}$ combined with its average value is closer to the industry standard (or $3.1 \mathrm{~mm}$ )

According to the actual working condition, the cam stroke is $12 \mathrm{~mm}$, the cam speed is $\mathrm{n}=72 \mathrm{r} / \mathrm{min}$, and the holding time is $5 \mathrm{~ms}$. 
Table1 Total high variance analysis table

\begin{tabular}{|c|c|c|c|}
\hline Sample cell & $n_{i}$ & $\overline{X_{i}}=\frac{1}{n_{i}} \sum_{i=1}^{n_{i}} X_{i}$ & $S^{2}=\frac{\sum_{i=1}^{n}\left(X_{i}-\bar{X}\right)^{2}}{n-1}$ \\
\hline $\begin{array}{llllll}3.16 & 3.20 & 3.20 & 3.12 & 3.14 & 3.18 \\
3.20 & 3.18 & 3.18 & 3.20 & 3.16 & 3.18 \\
3.18 & 3.18 & 3.20 & 3.20 & 3.18 & 3.16 \\
3.18 & 3.14 & & & & \end{array}$ & 20 & 3.176 & 0.000530526 \\
\hline
\end{tabular}

Table2 Side high variance analysis table

\begin{tabular}{|c|c|c|c|}
\hline Sample cell & $n_{i}$ & $\overline{X_{i}}=\frac{1}{n_{i}} \sum_{i=1}^{n_{i}} X_{i}$ & $S^{2}=\frac{\sum_{i=1}^{n}\left(X_{i}-\bar{X}\right)^{2}}{n-1}$ \\
\hline $\begin{array}{llllll}2.54 & 2.54 & 2.56 & 2.50 & 2.52 & 2.52 \\
2.48 & 2.58 & 2.54 & 2.58 & 2.54 & 2.50 \\
2.58 & 2.56 & 2.56 & 2.50 & 2.60 & 2.58 \\
2.54 & 2.56\end{array}$ & 20 & 2.544 & 0.00107789 \\
\hline
\end{tabular}

Average value and variance of the total cell height and the side height of the battery when the diameter of the film is sealed are obtained through sampling analysis.

Analyze the sealing film sealing the battery in the concave step angle and edge of the total height. As the following table:

Table3 Total high variance analysis table

\begin{tabular}{|c|c|c|c|}
\hline Sample cell & $n_{i}$ & $\overline{X_{i}}=\frac{1}{n_{i}} \sum_{i=1}^{n_{i}} X_{i}$ & $S^{2}=\frac{\sum_{i=1}^{n}\left(X_{i}-\bar{X}\right)^{2}}{n-1}$ \\
\hline $\begin{array}{llllll}3.12 & 3.10 & 3.14 & 3.12 & 3.12 & 3.14 \\
3.12 & 3.10 & 3.10 & 3.12 & 3.10 & 3.12 \\
3.14 & 3.14 & 3.12 & 3.14 & 3.14 & 3.12 \\
3.14 & 3.10 & & & & \end{array}$ & 20 & 3.122 & 0.0004 \\
\hline
\end{tabular}

Table4 Side high variance analysis table

\begin{tabular}{|c|c|c|c|}
\hline Sample cell & $n_{i}$ & $\overline{X_{i}}=\frac{1}{n_{i}} \sum_{i=1}^{n_{i}} X_{i}$ & $S^{2}=\frac{\sum_{i=1}^{n}\left(X_{i}-\bar{X}\right)^{2}}{n-1}$ \\
\hline $\begin{array}{llllll}2.50 & 2.52 & 2.54 & 2.50 & 2.52 & 2.50 \\
2.48 & 2.48 & 2.54 & 2.52 & 2.50 & 2.50 \\
2.52 & 2.54 & 2.54 & 2.50 & 2.48 & 2.50 \\
2.52 & 2.54 & & & & \end{array}$ & 20 & 2.512 & 0.000437895 \\
\hline
\end{tabular}

Through the above analysis, we can determine the angle of the sealing concave film angle of the quality of the standard: Edge height (or total height)and industry standards interval of $2.5 \mathrm{~mm}$ (or $3.1 \mathrm{~mm}$ ) are more close to each other, while the data variance is as small as possible. 
Table5 Total high mean and variance

\begin{tabular}{|c|c|c|}
\hline Sealing concave film & Mean value & Variance \\
\hline $\begin{array}{c}\text { Equal channel } \\
\text { angle }\end{array}$ & 3.176 & 0.000530526 \\
\hline Step angle & 3.122 & 0.0004 \\
\hline
\end{tabular}

Table6 Edge high mean and variance

\begin{tabular}{|c|c|c|}
\hline Sealing concave film & Mean value & Variance \\
\hline Equal channel angle & 2.544 & 0.001077895 \\
\hline Step angle & 2.512 & 0.000437895 \\
\hline
\end{tabular}

Through the above table, we can see that in average value which is close to the industry standard interval mean value and variance of the data, reducing angle sealing concave membrane numerical were better than angular diameter sealing.

\section{Conclusion}

It is concluded that the stress of the battery is smaller and force region is larger using step angle concave film sealing which is advantageous to the material's rebound control.

In the case of actual production conditions, the edge height and total height were measured in equal channel angular sealing film and the sealing concave concave angle adjustable film battery. By analyzing the variance of the data, the following conclusions are drawn:The numerical value of the radius of the sealing film has a significant effect on the total height and the height of the battery,and the step angle concave sealing membrane is more favorable for the consistency of the battery sealing quality.

\section{References}

[1]XU Ping-guo.Present situation and the development trend of button cell[J].Chinese Battery Industry,2002,7(3,4): 216-219.

[2]Li Tengfei.Research in remote monitoring and visual simulation technology of Lithium manganese button battery automatic sealing machine [D].TianJin: Tianjin university of technology,2011.

[3]Yu Mingyuan.Research on MT Secondary coin cell[D].TianJin: Tianjin university,2007.

[4] WANG Benri. The Springback Compensation for Car Stamping Die Surface [J]. Journal of Shanghai Electric Technology,2009,2(4): 1-4.

[5] Z T Zhang, D Lee. Development of a New Model for Plane Strain Bending and Springback Analysis[J].Journal of Materials Engineering and Performance, 1995, 4(3):291-300.

[6] WANG Li-min. Finite element analysis based on solidworks automatic plastic cups filling and sealing machine [J]. Machinery,2010,06:63-66.

[7]ABAQUS.Using ABAQUS finite element analysis software applied in mechanical industry [J].Machinery Industry Informatization and Network,2006,02:44-45. 
